\title{
La réception ambiguë du terme de genre dans la gestion médicale de l'intersexuation en France (1955-1975)
}

\begin{abstract}
Michal Raz
[Résumé] Cet article pose la question de la réception du terme gender au sein de la sphère médicale avant sa réappropriation féministe, et de son usage en tant que concept psychologique de régulation médicale des corps (inter)sexués. En traçant une histoire intellectuelle du concept - depuis sa naissance aux États-Unis dans les années 1950, et jusqu'à son importation, application et interprétation en France, au sein des équipes médicales et psychologiques spécialisées dans la prise en charge des personnes intersexuées -, l'auteure interroge la façon dont le gender a traversé I'Atlantique. En étudiant ses traductions discursives et pratiques, elle montre comment ce concept-outil constitue la clé de normalisation précoce du sexe atypique.. Mots-clés : Intersexualité, genre, médecine, France/Etats-Unis, psychanalyse.

[Abstract] This article aims to analyze the reception of the term gender within the medical sphere before its feminist reappropriation, as well as its use as a psychological concept serving the medical regulation of (inter)sexual bodies. By tracing an intellectual history of the concept - from its birth in the United States of the 1950s, to its importation, application and interpretation in France, among the medical and psychological teams specialized in the management of intersex people -, the author examines the ways gender has crossed the Atlantic. By identifying its discursive and practical translations, she shows how this concept constitutes a key to the early normalization of atypical sex.
\end{abstract}

Keywords: Intersexuality, gender, medicine, France/USA, psychoanalysis.

"Le genre, aujourd'hui tout le monde a l'air de découvrir ça en France, mais ça fait... en 1960 quand je suis allé là bas [à l'hôpital Johns Hopkins], c'était au centre de la réflexion de John Money. »

R. Rappaport ${ }^{1}$

Dans son article "Le gender est-il une invention américaine ?», Karen Offen démontre qu'il existe, au moins depuis le XVIII ${ }^{\mathrm{e}}$ siècle, « un usage français du terme

\footnotetext{
${ }^{1}$ Professeur de biologie du développement et de la reproduction, ancien chef de l'unité d'endocrinologie pédiatrique et du diabète à l'hôpital Necker-Enfants malades, et un des fondateurs de l'endocrinologie pédiatrique en France (entretien, février 2014).
} 
'genre', qui spécifie dans le vocabulaire sociopolitique - notamment féministe - la construction sociale et culturelle des sexes » (Offen, 2006, 291). Par conséquent, le rejet du concept en France, y compris par de nombreuses féministes, considéré comme une invention américaine et intraduisible, ne peut se comprendre que par sa contextualisation historique et politique (Fassin, 2008), et l'existence d'une «rhétorique binationale (France versus États-Unis)» (Kraus, 2005, 168). D'ailleurs, l'étymologie du mot révèle que le terme anglais est dérivé du verbe « gendrer » (engendrer) de l'ancien français, provenant du terme latin genus, generis (origine, naissance). En anglais comme en français, le mot renvoyait traditionnellement à la grammaire (Haig, 2004), avant que le terme gender ne soit conceptualisé et distingué du sexe par des psychologues états-unien-ne-s des années 1950 et 1960, travaillant dans des services médicaux de prise en charge de personnes intersexuées ${ }^{2}$ et transgenres/transsexuelles (Löwy, 2003). Dans les deux langues, il s'agit donc d'un terme polysémique, ayant une origine commune, de sorte que le refus du concept de genre n'est pas une pure question de traduction linguistique. Si de multiples travaux se sont intéressés à cette réticence française à l'égard du genre dans le contexte de sa conceptualisation féministe, dès les années 1970 et surtout 198033 (Fougeyrollas-Schwebel, 2003; Möser, 2013; Varikas, 2006), il s'agirait ici plutôt de poser la question de la réception du terme gender au sein de la sphère médicale avant sa réappropriation féministe, et de son usage en tant que concept psychologique de régulation médicale des corps sexués. Ces questions nous intéressent dans la mesure où elles permettent d'historiciser les usages et significations du genre en effectuant un retour réflexif sur sa propre trajectoire intellectuelle et disciplinaire. Ainsi peut-on commencer à tracer une histoire intellectuelle dans le contexte français des réflexions et interventions médico-psychologiques sur les individus défiant la «normalité » du sexe/genre.

En effet, loin d'être une question absente en France, le genre existait déjà dans les textes médicaux traitant de l'intersexuation, terrain de recherche particulièrement intéressant à cet égard puisqu'elle a été à la fois le lieu où médecins et psychologues ont développé l'idée du genre, et un champ de recherche fertile pour les critiques féministes de la différence sexuée, notamment aux États-Unis (Fausto-Sterling, 2012[2000] ; Kessler \& McKenna, 1978). Ces critiques ont élaboré une conception du genre distincte de celles des médecins, en utilisant le genre comme un outil critique qui sert non seulement à distinguer le sexe biologique et le sexe psychologique, mais également à saisir le sexe comme une construction sociale et biomédicale du féminin

\footnotetext{
${ }^{2}$ Selon une définition large, un individu intersexué est un individu ayant un sexe atypique, qui ne se laisse pas facilement classer dans la binarité masculin/féminin. Cela peut concerner les chromosomes, les gonades, les organes génitaux etc.

${ }^{3}$ Chez les féministes françaises, c'est probablement Christine Delphy qui revendique et investit le terme de genre en premier (Delphy, 1977, 1981).
} 
et du masculin qui passe par les corps et les comportements (Kraus et al., 2008). Cette re-signification du terme remet en cause la bicatégorisation par sexe (Kraus, 2000) et pense sa matérialité comme l'effet de normes sociales (Butler, 2009[1993]). Nous partageons cette conception qui constitue le point de départ de notre généalogie des savoirs sur le sexe atypique en France. Cette généalogie se limite ici à la sphère conceptuelle et terminologique, sans nier qu'indubitablement, la production des savoirs et des pratiques régulant le sexe résulte aussi bien des débats d'idées que des transformations matérielles et techniques (notamment de la chirurgie, de l'endocrinologie et de la génétique) qui rendaient pensable le concept de genre (Hausman, 1995). Ce travail interrogera donc plutôt le premier aspect tout en prenant en compte le développement historique du genre et son usage dans le domaine médical d'où il a émergé. Il propose ainsi une porte d'entrée pour mieux connaître les normes régulatrices du sexe ainsi que, plus généralement, la réflexion sur les (in)compréhensions des problématiques liées au genre en France.

Nous connaissons aujourd'hui le contexte dans lequel le concept a été développé dans les «cliniques de l'identité de genre " (Gender Identity Clinic), mais nous savons moins comment et par qui ces travaux ayant introduit la dissociation entre sexe et genre ont été importés, appliqués et interprétés par les médecins français dans le cadre des réflexions et des pratiques autour de l'intersexuation ${ }^{4}$. La question de cette réception française renvoie à une question, plus large que celle des individus ayant un sexe atypique, à savoir celle du mode de pensée et des pratiques médicales et psychologiques à l'égard du sexe dans la seconde partie du XXe siècle.

À travers l'analyse de publications médicales ${ }^{5}$ et des entretiens, cet article tentera de déceler les traductions discursives et pratiques du gender comme clé de diagnostic et de normalisation précoce du sexe atypique, et d'interroger la façon dont ces savoirs ont traversé l'Atlantique. Afin d'analyser l'importation, l'application et l'interprétation du concept, nous présenterons rapidement l'émergence du nouveau paradigme de prise en charge des intersexuée-s dans les années 1950, avant de nous demander si et comment ce paradigme, dont le concept emblématique fut le genre, a été traduit - linguistiquement et socialement - en France. Ce questionnement nous amènera à proposer quelques hypothèses sur les approches développées en France à l'égard de l'intersexuation mais aussi, plus largement, à l'égard du sexe et du genre.

\footnotetext{
${ }^{4}$ Le corpus étudié ici est plutôt celui de l'intersexuation et non pas du transsexualisme, qui a déjà été exploré, en particulier par Pierre-Henri Castel (2003).

${ }^{5}$ Les revues médicales analysées sont notamment: Pédiatrie, Annales d'endocrinologie, Gazette médicale de France, Archives françaises de pédiatrie, Chirurgie, Annales de pédiatrie, ainsi que des ouvrages et des thèses médicales sur le sujet.
} 


\section{Naissance d'un nouveau paradigme : les protocoles de Johns Hopkins}

En 1936, Lawson Wilkins fonde la première clinique d'endocrinologie pédiatrique à l'hôpital Johns Hopkins de Baltimore, clinique qui accueillera dès lors un grand nombre d'individus qu'on appelait «hermaphrodites»(Eder, 2012). Dans les années 1950, il forme une équipe de spécialistes qui entamera une grande étude sur les cas d'intersexuation. Cette équipe comporte des médecins mais aussi des psychologues dont le plus célèbre est John Money, sexologue et psychologue qui a rédigé une thèse sur le sujet et qui, en collaboration avec John et Joan Hampson, développe la première théorisation du genre dans une série d'articles publiés en 1955 (notamment Money et al., 1955a, 1955b).

Les travaux de cette équipe constituent un changement de paradigme dans la façon de penser le sexe et de traiter l'intersexuation. Les recommandations médicales sur ce sujet sont insérées dans le livre devenu classique que publie Wilkins en 1957. Cet ouvrage $^{6}$, ainsi qu'un article de 1955 (Wilkins et al,. 1955) témoignent de la rupture avec une tradition médicale états-unienne - et européenne - selon laquelle les opérations sur le sexe doivent être remises à l'âge adulte et aboutissent parfois à plusieurs changements de sexes consécutifs. Cette nouvelle conception se fonde sur une certaine conception de la sexuation, déployée par Wilkins dans un chapitre intitulé « Anomalies de la différence sexuelle », conception inspirée par le scientifique français Alfred Jost qu'il a rencontré à Johns Hopkins en 1949 (Jost, 1972). Wilkins y dénonce le fait de déterminer le sexe de l'enfant intersexué-e uniquement en fonction de son sexe génétique, gonadique ou hormonal. Selon ce que l'on désigne sous le terme «protocoles Hopkins » (Redick, 2005), il vaudrait mieux orienter le choix du sexe, sauf exception, en fonction de plusieurs facteurs, notamment de l'anatomie des organes génitaux externes et des possibilités chirurgicales qu'elle offre, "en particulier de la taille du phallus ou de la présence d'un vagin utilisable » (Wilkins, 1959, 285).

La conceptualisation du gender aux États-Unis émerge donc dans un moment de complexification des savoirs et des technologies du sexe (biologiques, génétiques, chirurgicaux ou psychologiques), caractérisé par la volonté d'établir des règles d'action claires, rapides et efficaces en séparant sexe biologique (sex) et social (gender). Ces nouvelles règles de conduite élaborées à la suite d'une étude de grande ampleur introduisent ainsi la prééminence du «sexe d'éducation» (rearing sex) sur celui des

\footnotetext{
${ }^{6}$ Il s'agit de la $2^{\mathrm{e}}$ édition de l'ouvrage Diagnostic et traitement des maladies des glandes endocrines de l'enfance et de l'adolescence, parue en anglais en 1957, dans laquelle le nouveau paradigme du genre apparaît. La $1^{\mathrm{e}}$ édition, datée de 1950, constitue le premier ouvrage d'endocrinologie pédiatrique et aborde la question de l'intersexuation encore selon l'ancien paradigme.
} 
chromosomes ou des gonades dans le développement précoce de l'enfant : « le genre masculin ou féminin - dans lequel un enfant est élevé, est le facteur dominant qui détermine la future orientation psychosexuelle» (Wilkins, 1959, 286), à condition bien sûr que ses organes génitaux externes soient conformes, ou deviennent conformes par les interventions, au sexe choisi. Or, pour que ces protocoles puissent être appliqués, il a fallu rendre à la fois possible et souhaitable le choix précoce du sexe, avant l'établissement du "rôle de genre» (gender role), considéré comme figé à partir de deux ans ${ }^{7}$, et au-delà duquel un changement induirait un risque de troubles psychologiques graves. Ainsi se trouvent justifiés les changements de sexe et les interventions de normalisation précoces par ce nouveau concept de genre forgé dans le cadre de recherches psycho-médicales sur les intersexué-e·s. Dès lors, en changeant de paradigme, on est passé d'un déterminisme à un autre : c'est le sexe - biologique - qui devient malléable, tandis que le genre, lui, devient presque irréversible au-delà de la petite enfance.

\section{Traductions et importations}

C'est ainsi dans la phrase citée ci-dessus, qui provient de la traduction française du livre de Wilkins (Wilkins, 1959) ${ }^{8}$, que le terme genre, dans le sens de gender role conceptualisé par Money et les Hampson, semble faire sa première apparition en français. Or, malgré cette traduction relativement rapide et la disponibilité du mot, le terme de genre était-il utilisé par les médecins spécialistes du sujet ? Et les recommandations thérapeutiques venant de l’hôpital Johns Hopkins étaient-elles reçues en France sans critique?

Si les travaux de Wilkins, comme ceux de Money et les Hampson, sont rapidement et largement lus, connus et cités en France dans les spécialités médicales concernées 9 , le concept qui sous-tend cette nouvelle pratique (distinction sexe/genre) n'est explicitement mentionné qu'occasionnellement. Certaines publications font état, dès la deuxième moitié des années 1950, de cette nouvelle approche selon laquelle l'orientation psychosexuelle n'est pas instinctive mais acquise par l'éducation, et appelle un choix

\footnotetext{
${ }^{7}$ Money et les Hampson parlent d'une période critique pour l'établissement du rôle de genre entre dix-huit mois et deux ans et demi (Money et al., 1955a), Certains auteurs ont retenu des âges limites pour le changement de sexe autour de deux ans, d'autres parlent de trois ans.

${ }^{8}$ Traduction de Jean Belaisch et André Cohen, gynécologues. La préface est d'Alfred GilbertDreyfus, endocrinologue. Cette traduction a probablement été réalisée en raison de l'importance de l'ouvrage pour l'endocrinologie et la pédiatrie et non pas pour ses apports concernant le développement sexuel.

${ }^{9}$ Notamment ceux de Wilkins concernant le traitement de l'hyperplasie congénitale des surrénales : Vague, 1956 ; Raffi et al., 1957 ; Neimann et al., 1956.
} 
précoce du sexe et des interventions chirurgicales (Bertrand \& Girard, 1958 ; Breton $e t$ al,. 1959; Decourt \& Guinet, 1962 ; Launay et al., 1958 ; Roget et al., 1957). Auparavant, la pratique voulait qu'on repousse les décisions irréversibles quant au sexe à l'adolescence ou à l'âge adulte. Or, comme en attestent des spécialistes parisiens, des transformations dans la prise en charge de ces enfants apparaissent :

[...] les soins de l'entourage, la façon différente dont on élève un garçon et une fille font qu'à l'âge de 2 ans environ le "sexe psychologique" » est déjà tellement fixé qu'il est très difficile et hasardeux d'entreprendre un changement de sexe. [...] II est donc indispensable d'arriver le plus tôt possible, dès les premiers mois, à un diagnostic précis du sexe dans lequel l'enfant devra être élevé, car on pourra alors prendre une décision ne tenant compte que des possibilités anatomiques et fonctionnelles réelles (Lelong et al., 1959, 613).

En dépit des nouveaux conseils de gestion précoce des cas, il était encore plus que fréquent, dans les années 1950-1970, que le premier diagnostic d'intersexuation ne soit fait qu'à un âge supérieur à la limite de deux ans. L'identification précoce de l'intersexuation est un phénomène relativement récent dû aux transformations sociales et technologiques de la deuxième moitié du XXe siècle, dont, entre autres, la médicalisation de l'accouchement et le développement des techniques d'exploration hormonale, génétique, radiologique et chirurgicale.

Dans ces travaux français des années 1955-1975 qui font référence au nouveau paradigme dans lequel le genre joue un rôle primordial, les termes utilisés sont variables, mais le mot genre, malgré sa traduction dès 1959, n'apparaît jamais. Si l'absence du terme ne dénote pas forcément une hostilité au paradigme, il pourrait néanmoins indiquer de possibles distanciations et critiques à l'égard du paradigme naissant qui s'imposera de façon internationale. On le retrouve parfois dans sa forme originale anglaise, gender role, comme dans un article écrit par un pédopsychiatre et un psychothérapeute, paru dans la revue lyonnaise Pédiatrie (Maer \& Bret, 1972), où est également utilisée une variante de l'expression fréquente "orientation psycho-sexuelle», lorsqu'ils affirment qu'il faudrait "orienter l'enfant vers une organisation psychosexuelle et sociale de garçon $»^{10}$. Cette expression apparaît dans de multiples publications médicales et psychologiques concernant les intersexuée-s (Demonsant, 1974; Neimann \& Fonder, 1967; Roget et al., 1957). D'autres expressions françaises ayant une signification proche du genre, comme le «sexe psychologique » (Launay et al., 1958; Lelong et al., 1959; Vassal, 1965) ou le « comportement somato-psychique » (Decourt \&

\footnotetext{
${ }^{10}$ Ils utilisent également l'expression « orientation sexuelle » pour désigner ce qu'on entend aujourd'hui par identité de genre.
} 
Guinet, 1962) apparaissent régulièrement dans les écrits et relèvent toutes du champ lexical du psychisme ${ }^{11}$.

D'ailleurs, l'importation des savoirs produits par l'équipe de Johns Hopkins vers la France ne se réduit pas à la lecture ou la traduction des textes. Comme dans d'autres phénomènes de circulation de savoirs scientifiques, elle se fait aussi au moyen de congrès internationaux, et de périodes de formation médicale spécialisée au sein d'équipes étrangères. Ainsi, plusieurs médecins français font un séjour au sein de l'équipe de Wilkins ${ }^{12}$ - voire toute une carrière, comme Claude Migeon -, notamment Jean Bertrand, devenu l'un des spécialistes de la question en France, et Raphaël Rappaport, qui fondera le service d'endocrinologie pédiatrique à l'hôpital Necker-Enfants malades (Migeon, 2014).

Ces échanges renforcent l'impression selon laquelle la diffusion de la nouvelle prise en charge des enfants intersexuéee-s, dans un environnement mêlant médecine et approche psychosociale, s'est faite assez rapidement vers la France. Néanmoins, la réflexion autour du genre rencontrait une organisation hospitalière distincte quant à la place des disciplines psychologiques. Certes, certains hôpitaux incluaient des pédopsychiatres - souvent d'approche psychanalytique - attachée.s au service pédiatrique (Saint Vincent de Paul à Paris, Debrousse à Lyon), mais le rôle de ceux-ci ne semble pas avoir été ni central dans les prises de décision cliniques ni dans les études scientifiques. En effet, dans la plupart des articles médicaux français sur l'intersexuation à cette période, la discussion psycho-sociale n'a que rarement sa place, au-delà de quelques lignes de conclusion, tandis qu'aux États-Unis le rôle des psychologues dans les cliniques de l'identité de genre a été primordial dans le développement de l'approche médicale.

Ainsi, la traduction de gender dans le livre de Wilkins n'est en quelque sorte qu'un accident collatéral, car les développements théoriques majeurs sur le concept, effectués par John Money et les Hampson, n’ont jamais été traduits, hormis un petit texte de Money qui paraît en français dans l'ouvrage collectif Le Fait féminin (Money, 1978). À l'inverse, l'autre auteur qui est connu pour ses écrits sur le gender, Robert Stoller, psychiatre et psychanalyste, a bien été traduit en français, quoique dix ans après la publication de Sex ef Gender, devenu un classique (Stoller, 1978[1968]). Dans cette traduction - sauf dans le titre, Recherches sur l'identité sexuelle - le terme gender est bien remplacé par celui de genre. Il en est de même pour un ouvrage ultérieur, Presentations

\footnotetext{
${ }^{11}$ Dans quelques publications d'avant 1955, les expressions utilisées sont «le point de vue psycho-somatique» (Cheymol et al., 1952), ou simplement «psychique » (Delore et al., 1953), un «pronostic sexuel » (Bernheim \& François, 1953), ou des «tendances profondes », psychologiques ou sexuelles (Decourt, 1953).

${ }^{12}$ Avec le service d'Andrea Prader à Zurich, celui de Wilkins était considéré comme le meilleur lieu de spécialisation en endocrinologie pédiatrique.
} 
of Gender (1985) traduit en 1989, avec pour titre Masculin ou féminin ? (Stoller, 1989) Les conclusions de Stoller sont ainsi traduites :

[...] les aspects de la sexualité que l'on appelle le genre sont essentiellement déterminés par la culture, c'est à dire appris après la naissance [...] ce processus culturel vient du milieu social, mais c'est par la mère que passe la connaissance, de sorte que ce qui atteint réellement l'enfant est la propre interprétation qu'elle donne habituellement des attitudes de la société. Plus tard, le père du petit enfant, ses frères et soeurs, ses amis, et progressivement l'ensemble de la société influent sur le développement de son identité (Stoller, 1978, 15).

Stoller est importé en France également parce qu'il travaille en grande partie sur la question du «transsexualisme » qui, comme le suggère Eva Rodriguez ${ }^{13}$, a suscité bien plus la curiosité des "psys» français que "l'hermaphrodisme», dans lequel ils ne voient pas un domaine de recherche. Dans son ouvrage La métamorphose impossible, Castel (2003) soutient également que la réticence à l'égard du genre serait liée à une tradition française psychanalytique se distinguant de la behavior therapy de Money

\section{Léon Kreisler - Les parents d'abord}

En France, vu le poids de la psychanalyse, ce sont les travaux de Stoller qui ont été le plus suivis, en retenant moins l'aspect sociologique du genre (milieu social, culturel etc.) que l'aspect parental du développement de l'identité psycho-sexuelle. Cette interprétation vient notamment de celui qui théorise en premier une approche psychiatrique en France à l'égard des intersexué·es : Léon Kreisler.

Kreisler, pédopsychiatre, travaille au sein de l'équipe spécialisée de l'hôpital pédiatrique de Saint Vincent-de-Paul à Paris dès les années 1960. Son expérience de prise en charge d'enfants ayant un sexe atypique et de leurs parents le conduit à rédiger un mémoire clinique monumental, Les intersexuels avec ambiguité génitale (Kreisler, 1970). Il y insiste sur la variété des cas individuels et prône donc une attitude plus flexible que celle qu'adopte Money quant au changement de sexe ${ }^{14}$. Selon lui, dans certains cas, des changements peuvent être faits après l'âge précoce de deux ans fixé par le protocole de Johns Hopkins. Une critique contre la directive américaine jugée trop

\footnotetext{
${ }^{13}$ Voir le dossier thématique établi par Eva Rodriguez, "Fabriquer le sexe, éduquer le genre », et notamment la partie 3 «De gender à genre : une traduction impossible ? » sur http://biosex.univ-paris1.fr/dossiers-thematiques.

${ }^{14}$ Un autre point qui distingue Money et Kreisler est l'approche statistique de Money, qui essaie de tirer des généralisations, contre une approche qualitative de Kreisler, qui va plutôt explorer la singularité de chaque cas. Ce serait une opposition entre «le pari français de l'empirisme clinique sur fond de psychanalyse » et « le risque de la généralité statistique pris par Money et à sa suite par les Américains » (Castel, 2003, 75).
} 
« rigide » a été en effet formulée non seulement par des psychiatres et psychanalystes, mais également par des médecins hospitaliers (Jeune et al., 1966; Gilbert-Dreyfus, 1972). Certains évoquent notamment leur réticence à l'égard de l'âge limite pour les changements de sexe, en grande partie parce qu'ils font souvent face à des individus âgés de 3, 7 ou 17 ans.

En 1972, dans un numéro spécial sur les états intersexuels dans la revue Pédiatrie, l'équipe lyonnaise critique cette limitation rigoureuse, et avance, sur la base de cas concrets, que la réussite psychologique ne dépend pas uniquement de l'âge de l'assignation, et qu'il «n'y ait pas de loi générale stricte concernant l'âge des changements de sexe. L'attitude dépend surtout des cas rencontrés, de la situation anatomique, des conditions familiales et en particulier du désir formel des parents. » (David et al., 1972, 888). La réticence à l'égard du genre est donc aussi une réticence à l'égard de la pratique qui en découlait. Dans une longue note de bas de page, Kreisler précise la terminologie qu'il utilise et écarte le mot « genre » au profit de la psychosexualité :

[...] Nous réservons les termes de 'sexe', 'sexualité', 'sexuel', aux faits d'ordre anatomique, physiologique et biologique. Pour les données d'ordre psychologique ou psychosociologique, nous suivons les habitudes de la littérature de langue française qui accole un terme supplémentaire et parle de psychosexualité, d'évolution psychosexuelle, d'orientation psychosexuelle, de rôle sexuel, d'identité sexuelle. Dans la littérature anglo-saxonne, on tend actuellement, dans un esprit de précision, à attribuer aux faits d'ordre psychologique ou psychosocial le terme : genre. On dit par exemple, rôle de genre (gender role : Money et Hampson), identité de genre (gender identity : Stoller). (Kreisler, 1970, 17) ${ }^{15}$

Malgré une impression de division culturelle entre approche américaine et française, de nombreux points d'entente existent entre les approches de Stoller et de Kreisler, dont la complicité professionnelle est d'ailleurs reconnue ${ }^{16}$. Les deux psychiatres d'inspiration psychanalytique insistent sur le rôle des parents, et en particulier sur ce-

\footnotetext{
${ }^{15}$ Kreisler (1970) définit l'identité sexuelle comme « le fait pour une personne de se reconnaître et d'être reconnue par autrui comme appartenant à un sexe ». Remarquons de plus que l'expression «identité sexuelle » (et non pas sexuée) crée une confusion entre la sexualité au sens de pratiques/orientations sexuelles, et l'identité féminine ou masculine. Plus tard, Colette Chiland, auteure française connue pour sa théorie psychanalytique de la transsexualité, précisera également qu'elle refuse le terme de genre. Dansson article sur I'identité sexuée de 1999 elle prévient : "Ce dont nous allons parler se dit gender identity dans le monde anglo-saxon. On pourrait dire en français "identité de genre". Mais nous ne pouvons pas utiliser en français le terme "genre" en composition avec la même facilité que l'anglais; gender role n'est pas immédiatement compris en français, où l'on a bon ou mauvais genre »(Chiland, 1999, 1251-2). Hormis les traductions, l'usage de genre en français est selon elle « barbare» $($ Ibid).

${ }^{16}$ Les deux ont par exemple échangé des manuscrits de leurs ouvrages avant leur publication, comme le raconte Kreisler dans son mémoire (1970).
} 
lui de la mère, dans le développement de la psychosexualité ou du genre de l'enfant. Cet accent mis sur la place des parents permet de mieux comprendre l'approche dominante en France : prendre en considération non pas tant le sentiment profond de l'enfant (va-t-il se sentir plutôt garçon ou plutôt fille ?), mais les opinions des parents (croient-ils que leur enfant est plutôt un garçon ou une fille ?). C'est la famille comme noyau premier des relations qui est toujours considérée comme primordiale, avant toutes les autres instances de socialisation comme la crèche, l'école, les pairs etc. Pour Kreisler, « l'intersexuel ambigu induit véritablement une maladie familiale » (Kreisler, 1970, 84). Certes, l'évaluation médicale des intersexuée-s contient un aspect psychologique, en détaillant le comportement de l'enfant (ses jeux, préférences etc.) et son « intelligence », mais c'est avant tout la personnalité des parents qui pèse sur la décision de la (ré)assignation du sexe, dans une approche au cas par cas.

Comme le dit encore Kreisler : « Dans cette incertitude il nous paraît que l'élément le plus positif peut être tiré de l'évaluation de la position des parents par rapport à la sexualité, la leur propre et celle de l'enfant » (Kreisler, 1970, 112), et de conclure que "l'identité psychosexuelle est fortement influencée par les attitudes inconscientes déclenchées chez la mère à la vue des organes génitaux » (Ibid, 119). Cette question de la responsabilité imposée aux mères dans la construction du genre de l'enfant pourrait faire l'objet d'une analyse approfondie, qui dépasse cependant cet article. Elle révèle en tout cas l'ancrage d'une vision psychanalytique particulière et sa façon de penser le sexe et le genre.

In fine, il semble que les professionnellle-s de la santé spécialistes des questions de sexualité n'ignoraient pas l'existence du concept de gender et de sa possible traduction en français. Ils ont consciemment choisi de le laisser de côté, pour des raisons linguistiques, pratiques et théoriques, mais aussi, sans doute, politiques et culturelles.

Néanmoins, le nouveau paradigme de gestion des cas d'intersexuation développé aux États-Unis a été rapidement discuté en France et son importation, au moins partielle, a été effectuée avec ou sans le terme de genre lui-même, en partie grâce à la circulation d'individus clés entre les deux côtés de l'Atlantique. En retraçant la réception du terme gender, ses traductions et ses absences, cet article entend entamer une analyse des positionnements du corps médical français vis-à-vis de la conceptualisation de la prise en charge et du traitement de l'intersexuation mais aussi vis-à-vis du développement de la personnalité et de la sexualité. Ainsi, les interprétations françaises ont critiqué le terme gender et un protocole jugé trop rigide, insuffisamment individualisé, et ont privilégié une évaluation au cas par cas des complexités culturelles, sociales et, principalement, familiales.

Ce dernier aspect semble jouer un rôle prédominant dans l'attitude médicale en France, profondément influencée par une approche psychanalytique focalisée sur les liens entre sexe, sexualité et relations intrafamiliales. Ainsi, le terme de psychosexualité traverse toutes les discussions sur les aspects psychologiques des enfants intersexuée-s et le développement de ce qu'on appelle aujourd'hui «identité de genre » est 
analysé avant tout sous l'angle des attentes, convictions et projections parentales. Ces discussions, parfois éloignées de la clinique elle-même, doivent être mises en regard de pratiques qui traduisent une double attitude: d'une part une adhésion au nouveau paradigme techno-conceptuel du diagnostic et des interventions précoces sur les organes génitaux des nouveaux né·e·s intersexué·e·s, appuyant une assignation légale, sociale et chirurgicale d'un sexe le plus tôt possible ; d'autre part, une défiance fréquente envers ce paradigme, que ce soit en raison des circonstances (âge tardif à la consultation), ou de particularités familiales («le désir formel des parents»), de sorte que les changements de sexe (à l'état civil et de façon chirurgicale), continuent à avoir lieu tout au long de l'enfance. Cette double attitude française des années 1950-1970 façonne le paysage conceptuel et les normes régulatrices du sexe agissant dans les cas d'intersexuation mais aussi au-delà. En ouvrant cette piste de réflexion, cet article appelle d'autres études des constellations médico-psychologiques à l'égard du sexe et du genre en France, de leurs présupposés, des concepts mobilisés et de leur mise en œuvre.

\section{Bibliographie}

Bernheim M., François R. (1953), «Syndromes cortico-surrénaux acquis de l'enfance », Pédiatrie, 8, 3, pp.357-403.

Bertrand I., Girard C. (1958), "La chromatine sexuelle et le diagnostic du sexe génétique dans les états intersexuels », Annales d’endocrinologie, 19, 2, pp.228247.

Breton A., Gaudier B., Ponte C., Poingt O., Delattre B. (1959), « Les états intersexués chez le nourrisson. Étude de trois observations», La semaine des hôpitaux, 14, 3, pp.1069-1083.

Butler J. (2009[1993]), Ces corps qui comptent : De la matérialité et des limites discursives du sexe, Paris : Éditions Amsterdam.

Castel P-H. (2003), La Métamorphose impensable : Essai sur le transsexualisme et l’identité personnelle, Paris : Gallimard.

Cheymol J., Jamain B., Henry R., Legros R. (1952), "Un cas d'ambiguïté sexuelle », Annales d'endocrinologie, 13, 2, pp.173-178.

Chiland C. (1999), «L'identité sexuée: Identité », Revue française de psychanalyse, 63, 4, pp.1251-1263.

David M., Mollard P., Daudet M., Lauras B. (1972), «L'ambiguité sexuelle dans le pseudo-hermaphrodisme féminin. À propos de 61 observations », Pédiatrie, 27, 8, pp.871-889. 
Decourt J. (1953), « Biotypologie sexuelle», Annales d'endocrinologie, pp.101-144.

Decourt J., Guinet P. (1962), Les états intersexuels, Paris : Maloine.

Delore P., Noel R., Perrin J., Guinet P., Blanc J. (1953), « Sur un cas d'hermaphrodisme vrai avec étude histologique de testicule et de l'ovaire », Pédiatrie, 8, 3, pp.458-462.

Delphy C. (1977), « Nos amis et nous. Fondements cachés de quelques discours pseudoféministes », Questions féministes, 1, pp.21-49.

Delphy C. (1981), «Le patriarcat, le féminisme et leurs intellectuelles », Nouvelles questions féministes, 2, pp.58-74.

Demonsant O. (1974), L'assignation du sexe dans le pseudo-hermaphrodisme masculin, thèse de médecine, Paris : Sodipe.

Eder S. (2012), « From 'following the push of nature' to 'restoring one's proper sex' cortisone and sex at Johns Hopkins's Pediatric Endocrinology Clinic», Endeavour, 36, 2, pp.69-76.

Fassin É. (2008), «L'empire du genre. L'histoire politique ambiguë d'un outil conceptuel », L'homme, 3, 4, pp.375-392.

Fausto-Sterling A. (2012[2000]), Corps en tous genres : La dualité des sexes à l'épreuve de la science, Paris : La Découverte.

Fougeyrollas-Schwebel D. (2003), Le genre comme catégorie d'analyse: sociologie, histoire, littérature, Paris : L'Harmattan.

Gilbert-Dreyfus A. (1972), Les Intersexualités, Paris : Presses Universitaires de France.

Haig D. (2004), « The inexorable rise of gender and the decline of sex: Social change in academic titles, 1945-2001 », Archives of sexual behavior, 33, 2, pp.87-96.

Hausman B. (1995), Changing sex: Transsexualism, technology, and the idea of gender, Durham : Duke University Press.

Jeune M., Peretti E., Mollard P., Hermier M., Laurent C., Couette Y. (1966), "Différenciation testiculaire unilatérale. (Dysgénésie gonadique mixte)», Pédiatrie, 21, 6, pp.699-708.

Jost A. (1972), Données préliminaires sur les stades initiaux de la différenciation du testicule chez le rat, Paris : Masson.

Kessler S., McKenna W. (1978), Gender : an ethnomethodological approach, Chicago: University of Chicago Press.

Kraus C. (2000), «La bicatégorisation par sexe à 'l'épreuve de la science' », in Löwy Ilana, Gardey D. (dir.), L'invention du naturel : Les sciences et la fabrication du féminin et du masculin, Paris : Éditions des archives contemporaines, pp.187-213. 
Kraus C. (2005), « Anglo-American Feminism made in France: crise et critique de la représentation », Cahiers du genre, 1, pp.163-189.

Kraus C., Perrin C., Rey S., Gosselin L., Guillot V., (2008), «À qui appartiennent nos corps ? Féminisme et luttes intersexes », Nouvelles Questions Féministes, 27, 1.

Kreisler L. (1970), «Les intersexuels avec ambiguité génitale. Étude psychopédiatrique », La psychiatrie de l'enfant, 13, 1, pp.5-127.

Launay C., Verliac F., Canlorbe P., Blanc A. (1958), « Problèmes d'avenir posés par un cas de pseudo-hermaphrodisme gynandroïde », Archives françaises de pédiatrie, 15, 1, pp.105-111.

Lelong M., Petit P., Canlorbe P., Sebaoun-Zucman S., Cendron J., Borniche P., Scholler R. (1959), «Les anomalies de la détermination sexuelle. À propos de 12 observations personnelles », Annales de pédiatrie, 16, pp.581-614.

Löwy I. (2003), «Intersexe et transsexualités : Les technologies de la médecine et la séparation du sexe biologique du sexe social », Cahiers du Genre, 34, 1, pp.81-104.

Maer M., Bret P. (1972), " Problème psychologiques et sociaux des changements de sexe. À propos d'une observation », Pédiatrie, 27, 8, pp.913-922.

Migeon C. (2014), «Lawson Wilkins and My Life: Part 3 », International Journal of Pediatric Endocrinology, Supplement 1, S4.

Money J., Hampson J., Hampson J. (1955a), «Hermaphroditism: recommendations concerning assignment of sex, change of sex and psychologic management», Bulletin of the Johns Hopkins Hospital, 97, 4, pp.284-300.

Money J., Hampson J., Hampson J. (1955b), «An examination of some basic sexual concepts: the evidence of human hermaphroditism», Bulletin of the Johns Hopkins Hospital, 97, 4, pp.301-319.

Money J. (1978), « Le transsexualisme et les principes d'une féminologie » in Sullerot E. (dir.), Le Fait féminin, Paris : Fayard, pp.223-233.

Möser C., (2013), Féminismes en traductions, Paris ; Archives contemporaines.

Neimann N., Fonder A. (1967), « L’hermaphrodisme », Pédiatrie, 22, 5, pp.499-549.

Neimann N., Lascombes G., Pierson M. (1956), «Le pseudo-hermaphrodisme par hyperplasie congénitale des surrénales », Archives françaises de pédiatrie, 13, 6, pp.596-612.

Offen K. (2006), « Le gender est-il une invention américaine ?», Clio. Femmes, Genre, Histoire, 24, pp.291-304. 
Raffi A., Mussini-Montpellier, Boineau N., Amsellem G. (1957), « Hirsutisme chez un nourrison de 10 mois par hyperplasie congénitale des surrénales », Pédiatrie, 12, 8, pp.865-870.

Redick A. (2005), "What Happened at Hopkins: The Creation of the Intersex Management Protocols », Cardozo Journal of Law \& Gender, 12, pp.289-296.

Rodriguez E., «Fabriquer le sexe, éduquer le genre », dossier thématique disponible en ligne : http://biosex.univ-paris1.fr/dossiers-thematiques.

Roget J., Douillet M., Beaudoing A., Revol M. (1957), «Pseudo-hermaphrodisme féminin sans hyperplasie surrénale », Pédiatrie, 12, 8, pp.859-864.

Stoller R. (1978[1968]), Recherches sur l'identité sexuelle à partir du transsexualisme, Paris : Gallimard.

Stoller R. (1989[1985]), Masculin ou féminin ?, Paris : Presses Universitaires de France.

Vague J. (1956), «Fréquence des intersexualités somatiques aux voies génitales normales », Annales d'endocrinologie, 17, 4, pp.485-489.

Varikas E. (2006), Penser le sexe et le genre, Paris : Presses Universitaires de France.

Vassal Jean. (1965), "Conduite à tenir devant une "ambiguité sexuelle” », Gazette médicale de France, 72, pp.113-132.

Wilkins L. (1959[1957]), Diagnostic et traitement des maladies des glandes endocrines de l'enfance et de l'adolescence, Paris : Maloine.

Wilkins L., Grumbach M., Van Wyk J., Shepard T. Papadatos C.(1955), « Hermaphroditism: Classification, Diagnosis, Selection of Sex and Treatment », Pediatrics, 6, pp.287-302 